\title{
Direct Measurement of Kinetic Energy Dissipation in Polysaccharide Conformation Transition at Single Molecule Level
}

\author{
S. Guo*, S. Jesse*, M.P. Nikiforov*, B.B. Akhremitchev**, S.V. Kalinin* \\ * Oak Ridge National Laboratory, Oak Ridge, TN 37831 \\ ** Department of Chemistry, Duke University, Durham, NC 27708
}

Polysaccharides are a group of biopolymers containing a variety of pyranose rings and glycosidic linkages. They play a vital role in many cellular functions, specifically in some stress-associated processes because of the conformation transition of pyranose ring structures under stress.[1] The force-induced conformation transition of polysaccharides has been intensively studied by the Atomic Force Spectroscopy (AFM) based single molecule force spectroscopy.[1-4] However, the kinetics of polymer conformation transition has been rarely studied because the high transition rate resulting from the low energy barrier among various conformations is beyond the traditional force spectroscopy detection frequency.

Here, we applied a dynamic single molecule force spectroscopy which combing the AFM force spectroscopy with the band excitation approach [5] to directly measure the kinetic energy dissipation in conformation transition of dextran molecule at single molecule level. The experiments were conducted in phosphate buffer solution ( $\mathrm{pH}$ 7.2) and to pick dextran molecules, a bare silicon tip was pressed down onto a clean glass substrate on which dextran molecules were sparely deposited. Dextran molecule can be absorbed onto the tip dring the tip-surface contact. Once molecule has been attached on probe during the probe withdraws, a band excitation signal will be applied on the cantilever. In the band excitation approach the cantilever is excited over a band of frequencies and the corresponding response is recorded simultaneously. As shown in panels (a), (b) and (c) of Figure 1 , when the probe pulls the dextran molecule, the force-separation curve is recorded, as well as the response of cantilever to the excitation at different frequencies during the pulling. We demonstrated that the kinetic energy dissipation is only visible at a certain high frequency band, suggesting the excitation frequency is comparable to the conformation transition rate. The kinetic energy dissipation of dextran conformation transition has been extracted from the cantilever response by functional fit (See panels d, e and $\mathrm{f}$ in Figure 1).

This dynamic single molecule force spectroscopy can be extended to characterize conformation transition of various polymers (such as DNA, peptides) and synthetic block copolymers.

\section{References}

[1] Marszalek, P.E., et al., Nature 396 (1998) 661

[2] Rief, M., et al., Science 275 (1997) 1295

[3] Rief, M., et al., Physical Review Letters 81 (1998) 4764

[4] Marszalek, P.E., et al., Proceedings of the National Academy of Sciences of the United States of America 99 (2002) 4278

[5] Jesse, S., et al., Nanotechnology 18 (2007) 
[6] This research has been conducted at ORNL's Center for Nanophase Materials Sciences sponsored by the Scientific User Facilities Division, Office of Basic Energy Sciences, U.S. DOE.
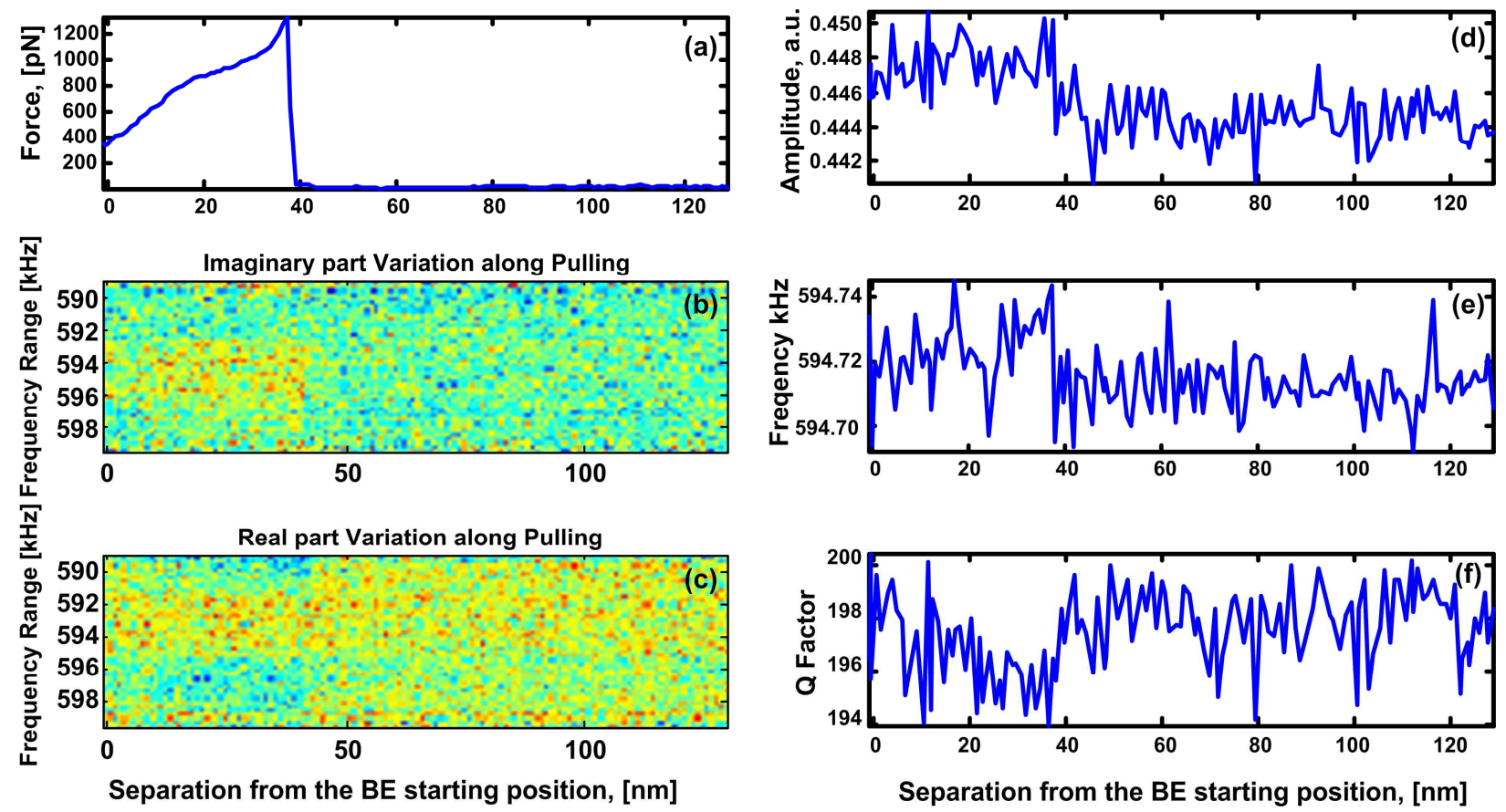

FIG. 1. (a) Force-separation curve when the AFM probe pulls a dextran molecule and conformation transition occurs when the pulling force increases to $\sim 900 \mathrm{pN}$. (b) Variation of the imaginary part of response along the pulling. (c) Variation of the real part of response along the pulling. (c) The response amplitude has been fitted by the Simple Harmonic Oscillator (SHO) model and amplitude (d), frequency (e) and Q factor (f) have been extracted. 